\title{
Von der Routine zur Resignation?
}

\author{
B. Gurtner
}

Auf gewundenen Pfaden oder in der Direttissima haben viele von uns die Gipfel oder wenigstens die Hochebene des beruflichen Könnens erreicht. Oft verliefen die Lernkurven auf dem Buckel der Patienten oder schlimmer noch in ihren Eingeweiden. Zwar übten wir als Studenten am Phantom des weiblichen Beckens und an ledernen Föten die geburtshilflichen Handgriffe und den korrekten Gebrauch der Zangen. Das waren aber Fertigkeiten, welche die meisten nach dem Staatsexamen nie mehr benötigten. Nützlicher war es, Wundnähte an Schweinehaut zu erproben oder lebensgrosse Reanimationspuppen herzlich zu massieren und zu intubieren. Je nach Fachgebiet kamen später weitere Trainings mit simulierten Einsätzen hinzu, für manche Prozeduren galt aber das berüchtigte «see one - do one - teach one».

Inzwischen sind die Ausbildungswege genauer abgesteckt worden, und man muss in regelmässigen Abständen nachweisen, dass man die Fähigkeit bewahrt hat, schwierige Situationen zu meistern. Es dürfte heute kaum mehr vorkommen, dass berühmte Chirurgen daran gehindert werden müssen, in ihrem Altersstarrsinn operativ missetätig zu sein. $\mathrm{Zu}$ wenig beachtet aber wird die schon in jüngeren Jahren lauernde Gefahr, wenn zunehmende Routine zu abnehmender Aufmerksamkeit oder zu unverantwortlichen «mutigen» Entschlüssen verleitet. Anfänger machen Fehler, obwohl sie aufpassen, ihren Chefs passieren sie, wenn sie als Könner ausnahmsweise einmal nicht voll konzentriert sind. Die in der Aviatik bewährten Checklisten, welche auch die erfahrensten Flugkapitäne vor jedem Start abzuhaken haben, finden erst langsam Eingang in die Medizin und werden noch oft als Zumutung empfunden.

Ein hochspezialisierter Herzchirurg hat nach vielen Jahren erfolgreicher Tätigkeit die weiten Horizonte im engen Operationsfeld vermisst und ist Lastwagenfernfahrer geworden. Auch den Grundversorgern droht midlive eine Ernüchterung oder gar Langeweile, die Faszination des Berufes kann schwinden und damit auch die ärztliche Ausstrahlung und Zuverlässigkeit. Erlebnisse häufen sich, die zeigen, wie hinfällig therapeutische Erfolge oft sind und wie sie durch
Unvernunft der Behandelten zunichte gemacht werden. Da gleitet man unmerklich ab in eine milde Resignation oder gar in einen patientenverachtenden Medizynismus, der sich nach einem Missgeschick damit tröstet, das ohnehin alle einmal sterben müssen.

Kollege Anton Tschechow, der vor 100 Jahren einer Lungentuberkulose erlag, nannte die Medizin seine rechtmässige Gattin und die Literatur seine Geliebte, zu der er flüchtete, wenn ihn der Beruf erschöpfte. In der Erzählung «Krankensaal Nr. 6» zeichnet er den ausgelaugten Arzt Andrej Jefimytsch Ragin, der an einem schmutzigen Provinzspital tätig oder - besser gesagt - in stoischer Unbekümmertheit untätig ist, weil er angesichts des sozialen Elends und der Vergänglichkeit jeder menschlichen Existenz am Sinn medizinischer Massnahmen zweifelt. In den immer kürzer gehaltenen Sprechstunden nerven ihn die unverständigen Patienten und er mag seine eigenen Fragen, die er ihnen seit über 20 Jahren stellt, schon gar nicht mehr hören.

In langen philosophischen Gesprächen mit dem zwangshospitalisierten Querkopf Iwan Dmitritsch kommt vieles hoch, was Tschechow selbst bedrückt hat. Als junger Arzt hatte er die Gefangenenlager in Sachalin besucht, Hungersnöte kennengelernt und Choleraepidemien bekämpft. Er sah die grosse Zukunft der modernen Medizin voraus, hat sich aber gefragt, was die wissenschaftlichen Fortschritte wert sind, wenn sie bei vielen Menschen nur ein Leben verlängern, das von Mühsal, Dummheit, Streit und Süchten geprägt ist.

Burn-out würde man dem heute sagen, der Ofen ist kalt geworden. Bei Tschechow, der sich dagegen gewehrt hat, als Pessimist verkannt zu werden, war es eher ein langsames Burningdown am Ende eines kurzen, aber sehr produktiven Lebens. Auch seine letzten Werke, die er sich - bereits todkrank - abgerungen hat, spenden noch etwas Licht und Wärme und wenden sich gegen die kalte Routine oder traurige Resignation, welche der tägliche berufliche Kontakt mit menschlichem Elend auslösen kann. Der Klappentext der literarischen gelben Taschenapotheke [1] bringt es auf den Punkt: «Er blieb den Leidenden in Liebe zugetan.» 